\title{
Lending Decisions with Limits on Capital Available: The Polygamous Marriage Problem
}

\author{
Mee Chi So*1 $\quad$ Lyn C. Thomas ${ }^{1} \quad$ Bo Huang $^{2}$ \\ ${ }^{1}$ Southampton Management School, University of Southampton, Southampton SO17 1BJ, United Kingdom. \\ ${ }^{2}$ School of Finance, Renmin University of China, NO.59 Zhongguancun Street, Haidian District, Beijing, \\ P.R.China, 100872
}

\begin{abstract}
In order to stimulate or subdue the economy, banking regulators have sought to impose caps or floors on individual bank's lending to certain types of borrowers. This paper shows that the resultant decision problem for a bank of which potential borrower to accept is a variant of the marriage/secretary problem where one can accept several applicants. The paper solves the decision problem using dynamic programming. We give results on the form of the optimal lending problem and counter examples to further "reasonable" conjectures which do not hold in the general case. By solving numerical examples we show the potential loss of profit and the inconsistency in the lending decision that are caused by introducing floors and caps on lending. The paper also describes some other situations where the same decision occurs.
\end{abstract}

Keywords: Dynamic programming; Markov processes; Consumer credit lending 


\section{Introduction}

Financial regulators in several countries have sought to rein in or alternatively encourage lending to borrowers by putting caps or floors on the amount a bank can lend over a given time period. Countries such as China, India or Turkey have imposed caps on lending for a number of years. More recently, the UK government also sought to have an annual floor on a bank's lending to small and medium sized enterprises (SMEs). Imposing such regulations on lending will change the decisions on whom to lend to as well as making the lending less profitable for the bank and in consequence leading to inefficient lending. It can also mean there is unfairness in the lending as the accept/reject decision by the bank depends on how much capital is still available before the limit is reached and how long it is until the end of the lending restriction horizon. The objective of this study therefore is to identify the impact of putting caps/floors on inefficiencies and unfairness by solving the lender's accept/reject decision problem optimally.

This paper develops a set of Markov Decision Processes (MDP) models which address the lending problem with constraints on the total capital lent. From these, it is possible to investigate the optimal lending policies and how they differ in which borrowers are being accepted and the lender's total profitability compared with the optimal policy when there is no restriction on capital. The models themselves have a flavour of multiple choice secretarial or marriage problems, or the house hunting problem but are quite different in the objective to be optimized and the information available to the decision maker. In this problem the objective is to maximise the total profit to the lender rather than maximising the probability of choosing the $\mathrm{k}$ most profitable borrowers. When a potential borrower requested a loan, the lender is told two characteristics - the size of the loan requested and the probability of the borrower not defaulting on the loan. The latter of these is given by a credit score. The form of the optimal policy is to accept a borrower if their probability of non-default is above a certain value which translates into the credit score being above some cut-off score. However, unlike the traditional problem with unlimited capital available, this cut-off level will vary depending on the capital still available and the time until the lending restrictions end. These models show how significant is this unfairness to borrowers and also the drop in the lender's profitability that these capital restrictions cause. 
In section 2, we review the literature outlining the restrictions on consumer lending, the basic consumer lending model and the related literature on the secretary problem. In section 3 we define the lending model with a cap on the amount of capital that can be lent in a given time period. This is a Markov Decision Process model and we describe the optimality equation and the form of the optimal policy. We also suggest two other policies including the optimal policy if there is no cap on the capital. Section 4 describes a discrete state space simplification of the model. This allows us to calculate several numerical examples including some that prove to be counter examples to reasonable conjectures concerning the optimal policy. It also means we can evaluate the difference between the optimal capped and uncapped policies and so address the fairness and profit sub optimality of the former. Section 5 looks at the problem where there is a floor on the capital that must be lent. This has been advocated by several governments particularly for bank lending to SMEs. It turns out that this problem leads to essentially the same Markov Decision Process model as the lending problem with a cap on the capital. We write the original problem with general cost functions though we describe the problem in the text in terms of the lending problem. In section 6 we describe three other problems which can lead to the model of section 3. We draw some conclusions in the final section.

\section{Literature Review}

In the last decade, regulators in several countries have sought to improve the economy by putting floors or caps on the annual level of an individual bank's lending to consumers or small businesses. The Central Bank of China set annual limits on the new lending by individual banks (Bloomberg, 2012) for a number of years. In 2011, Turkey's bank regulator penalised banks that exceeded a limit on consumer lending. On the other hand, some governments sought to increase the lending to companies, particularly small and medium sized (SMEs) ones by putting floors on each bank's lending in that sector. The most notable of these was the agreement between the UK government and four international banks enshrined in the Merlin Project (Bank of England, 2012). The Bank of India had already in 2006 imposed a floor on banks' lending in this sector (Reserve Bank of India, 2009).

The impacts of these restrictions on the individual lending decisions are modelled in this paper. Opportunities to invest arrive according to a random process and are described by two characteristics - the size of the resource required and the probability of the opportunity being 
successful. The distribution of these over the whole set of possible opportunities is known but not their value on a specific opportunity until it appears. If the opportunity is rejected it cannot subsequently be accepted. The objective is to maximise the total expected profitability of the opportunities accepted assuming that there is a fixed time horizon for investment and a limit on the resource available. This has the flavour of a number of classic decision problems but does differ from them in several aspects.

In the secretary problem, sometimes called the marriage problem and reviewed by Freeman (1983) and Ferguson (1989), each opportunity only has one characteristic whose probability distribution is not known. The objective is to maximise the probability of choosing the opportunity with the maximum characteristic value. There have been many variants of this problem including Smith (1975) who allows the opportunity to refuse to be accepted and Yoshidi (1984) who allows a change point in the probability distribution. Preater (1994) looked at the multiple-choice problem where one can choose $k$, where $k>1$, opportunities and the objective is to maximise a utility function of the ranks of the $k$ opportunities chosen. Bateni et al (2010) reviewed a number of multiple choice secretarial problems where the objective is to maximise the expectation of a submodular function based on the skills of the secretarial group chosen. Extension of the classical problem has also been done by Chun (1999) who looks at the decision problems when there are more than one choices are available or by Stein et al. (2003) who compares the computational complexity of three heuristic solution approaches.

In the house hunting problem (Derman and Sachs, 1960; Ferguson and Klass, 2010), there is a cost of examining each opportunity. The value of the characteristic of each opportunity is i.i.d. and the distribution is known. The objective is to stop at an opportunity so the total value of the characteristic of that opportunity less the examination costs is maximised.

Choice problems closer to that considered in this paper are the sequential allocation problems first introduced by Derman, Lieberman and Ross (1975). In these problems, opportunities arrive according to a random process over a finite time horizon. The decision maker has a limited amount of resource to invest and the profit from each opportunity invested in is the same non-decreasing function of the investment level. The difference with the problem considered in the following sections is that the amount to invest is a decision by the investor whereas with us it is a characteristic of the opportunity. Moreover, our opportunities have 
different profits since these depend on the probability of repayment of the opportunity. Prastacos (1983) extended this problem by allowing the profit to depend on the quality of the opportunity. However, the decision is still how much to invest rather than whether or not to invest the amount required, which is the case in our problem.

Another way of thinking about the problem is as a dynamic stochastic knapsack problem (Kleywegt and Papastavrou, 1998). In this problem, items arrive to be loaded on a container of fixed size (the resource). The size and value of each item is unknown until the item arrives although the distribution of sizes and values is known. There is a holding cost per unit time until it is decided to dispatch the shipment and there is a value for any unused capacity. The objective is to maximise the expected overall value dispatched less the costs. The decisions are whether to accept an item and when to dispatch the shipment. Setting the holding cost and the value of unused capacity to zero would lead to a problem similar to the simplest one considered in this paper. Kleywegt and Papastavrou (1998) look only at the case where all items are the same size, which is akin to the case in Theorem 2 of this paper. Kleywegt and Papastavrou (2001) looked at the case of variable sizes but when there is no deadline on when the shipment needs to be sent. They also looked at the problem with a shipment deadline and found under what conditions the optimal policy had some monotone properties, something that did not hold in general. Subsequent works (van Slyke and Young, 2000; Zhuang et al., 2012) have modified the problem to deal with the conditions that arise in yield management. The difference to the problem considered in this paper is that our opportunities have a probability of success and so the optimal policy is based on a cut-off on the riskiness of the opportunity rather than a control limit policy on the size of the loan. Moreover, the boundary conditions of fully funding or partially funding the opportunities that edge over the constraint cannot be used in the knapsack approach. Similarly, the floor problem we discuss does not fit into the knapsack approach.

\section{Lending Model with Ceiling on Capital Available}

Opportunities arrive according to a Poisson process with arrival rate $\lambda$. Think of these as borrowers applying for a loan. Each opportunity $i$ requires an investment of $x_{i}$ and has a probability $p_{i}$ of a successful outcome with a reward of $S$ per unit invested if successful and a loss of $-\mathrm{F}$ per unit invested if a failure.. The expected reward is then $x_{i}\left(p_{i} S-\left(1-p_{i}\right) F\right)$. For the lending problem to consumers, the model found in Thomas (2009) suggests 


$$
S=\left(r-r_{F}\right), F=\left(l_{D}+r_{F}\right)
$$

where $r$ is the interest rate charged on the loan; $r_{F}$ is the risk free interest rate at which the lender can borrow the money that is being subsequently lent; $l_{D}$ is the loss given default on the loan, which is the percentage of the loan that is finally lost at the end of the collections process. The lender does not know in advance the required investment level or risk probability of each loan but does know that the overall distribution of $(x, p)$ is given by a density function $f(x, p)$.

When the borrower arrives the lender finds out the $x$ and $p$ for that applicant. The latter is usually expressed as their credit score. The decision maker has to decide when each opportunity arrives whether to accept it or reject it but is only allowed to invest $L$ in total in the time horizon $T$ of interest. The aim is to maximise the expected reward in this period.

Let $V(l, t, x, p)$ be the future expected reward given an opportunity has just arrived with $t$ more of the period to go and there is $l$ of the investment capital still available while the opportunity that has arrived requires an investment of $x$ and has a probability of success of $p$.

Let $R(l, t)$ be the optimal expected future reward if there is still $t$ to go and $l$ investment capital available and no opportunity currently available.

Similarly, let $W(l, t)$ be the optimal expected future reward with $t$ to go, and $l$ investment capital still available when there is an opportunity available but whose $x$ and $p$ is not yet revealed. These are connected by the following dynamic programming optimality equations:

$$
\begin{aligned}
& V(l, t, x, p)=\max \left\{\begin{array}{c}
R(l, t): \text { reject } \\
x(S p-(1-p) F)+R(l-x, t): \text { acccept }
\end{array}\right. \\
& \text { where } R(l, t)=\int_{0}^{t} \lambda e^{-\lambda s} W(l, t-s) d s \\
& \text { and } \mathrm{W}(l, t)=\int_{0}^{1} d p \int_{0}^{\infty} d x f(x, p) V(l, t, x, p)(3)
\end{aligned}
$$

Theorem 1: The optimal policy is accept the opportunity if 


$$
\begin{aligned}
& p \geq p^{*}(l, t, x) \text { where } \\
& p^{*}(l, t, x)=\frac{1}{S+F}\left(\frac{R(l, t)-R(l-x, t)}{x}+F\right)
\end{aligned}
$$

Proof. The proof follows trivially from (2).

There are three variants of this model depending on what the lender is allowed to do when the next opportunity, if accepted would break the investment limit. This is the situation in the state $(l, t, x, p)$ where $l>0>l-x$. The three possibilities are:

a) Allow overshoot of the limit so the loan is fully funded

$$
V(l, t, x, p)=\max \left\{\begin{array}{c}
R(l, t) \\
x(S p-(1-p) F
\end{array}\right\}
$$

b) Allow the loan to be partially funded

$$
V(l, t, x, p)=\max \left\{\begin{array}{c}
R(l, t) \\
l(S p-(1-p) F
\end{array}\right\}
$$

c) Loan is not funded at all

$$
V(l, t, x, p)=\max \left\{\begin{array}{c}
R(l, t) \\
0
\end{array}\right\}
$$

It follows from this that the decision rule (4) changes in the situation when $x>l>0$ to

$$
\begin{aligned}
& p^{*}(l, t, x)=\min \left\{\frac{1}{S+F}\left(\frac{R(l, t)}{x}+F\right), 1\right\} \text { fully funded } \\
& p^{*}(l, t, x)=\min \left\{\frac{1}{S+F}\left(\frac{R(l, t)}{l}+F\right), 1\right\} \text { partially funded } \\
& p^{*}(l, t, x)=1 \quad \text { not funded }
\end{aligned}
$$

These follow since $R(l, t)$ must be 0 if $l \leq 0$. In the subsequent work, Initially we concentrate on the results in the fully funded case because (5) is of the same form as (2) provided we set $R(l, t)=0$ for all $l \leq 0$. We will discuss the other two cases when they give different results. 
We can prove result about $V, R, W$ and $p^{*}$ by using value iteration, which is a standard approach to solving Markov Decision Processes (Bauerle and Rieder, 2011). We use the following value iteration equations to help prove such results:

$$
\begin{aligned}
& V_{n+1}(l, t, x, p)=\max \left\{\begin{array}{c}
R_{n}(l, t) \\
x(S p-(1-p) F)+R_{n}(l-x, t)
\end{array}(9)\right. \\
& \text { where } R_{n}(l, t)=\int_{0}^{t} \lambda e^{-\lambda s} W_{n}(l, t-s) d s \\
& \text { and } \mathrm{W}_{\mathrm{n}}(l, t)=\int_{0}^{1} f(x, p) d p \int_{0}^{\infty} V_{n}(l, t, x, p) d x(10) \\
& \mathrm{V}_{0}(l, t, x, p)=W_{0}(l, t)=R_{0}(l, t)=0
\end{aligned}
$$

\section{Lemma 1:}

i) $V_{n}(l, t, x, p), R_{n}(l, t)$ and $W_{n}(l, t)$ are non-decreasing in $n$ and hence converge to $V(l, t, x, p), R(l, t)$ and $W(l, t)$;

ii) $V(l, t, x, p), R(l, t)$ and $W(l, t)$ are all non-decreasing in $l$ and in $t ; V(l, t, x, p)$ is nondecreasing in $p$.

\section{Proof:}

i) That $V_{n}(l, t, x, p), R_{n}(l, t)$ and $W_{n}(l, t)$ are all non-decreasing in $n$ follows by induction on $n$ where the critical induction step is recognising that

$$
\begin{aligned}
& V_{n+1}(l, t, x, p)-V_{n}(l, t, x, p)=\max \left\{\begin{array}{c}
R_{n}(l, t): \\
x(S p-(1-p) F)+R_{n}(l-x, t)
\end{array}-\max \left\{\begin{array}{c}
R_{n-1}(l, t): \\
x(S p-(1-p) F)+R_{n-1}(l-x, t)
\end{array}\right.\right. \\
& \geq \min \left\{\begin{array}{c}
R_{n-1}(l, t)-R_{n-1}(l, t): \\
R_{n-1}(l-x, t)-R_{n-1}(l-x, t)
\end{array} \geq 0\right.
\end{aligned}
$$

So the iterates of $V, R$ and $W$ are all non-decreasing and bounded by $l$. And so converge to bounded functions. Hence from Puterman (1994) they converge to the solution of equations (2) to (3).

ii) Again this is proved using induction on the iterates of value iteration. Induction trivially holds at $n=0$ since $W_{0}(l, t)=R_{0}(l, t)=V_{0}(l, t, x, p)=0$. Assume the result holds for $V_{n}(\ldots, \ldots$,$) then by (10)$ and (9) it trivially holds for $W_{n}(.,$.$) and for R_{n}(.,$.$) . Lastly from$ (8) it then holds for $V_{n+1}(., \ldots,$.$) and the induction step is complete. The impact of p$ on $V(., \ldots,$.$) is trivial from (2).$ 
One might expect results concerning the capital available and the time available, namely the more capital available the higher has to be the quality of the applicants accepted. Similarly one might expect that the more time available the higher an applicant's quality has to be so as to be accepted. However, as a counter example given in the next section shows, the result concerning capital is not true in the general case. The relationship between capital available and acceptance decision is true only if all applicants apply for the same amount of capital. This is what the next theorem implies.

Theorem 2: Suppose $\int_{0}^{1} f\left(x_{0}, p\right) d p=1$, so all applicants only apply for loans of $x_{0}$ then i) $R(l, t), W(l, t)$ and $V\left(l, t, x_{0}, p\right)$ are all concave in $l$;

ii) $p^{*}\left(l, t, x_{0}\right)$ is non-increasing in $l$.

\section{Proof.}

i) If every loan application is for $x_{0}$, then the capital available dimension of the state space becomes $\left\{L-m x_{0}\right.$ where $\left.0 \leq m \leq\left|\frac{L}{x_{0}}\right|+1\right\}$. So to prove concavity in $l$ it is enough to show that

$$
\begin{aligned}
& V\left(L-m x_{0}, t, x_{0}, p\right)-V\left(L-(m+1) x_{0}, t, x_{0}, p\right) \\
\geq & V\left(L-(m-1) x_{0}, t, x_{0}, p\right)-V\left(L-m x_{0}, t, x_{0}, p\right)
\end{aligned}
$$

If $V(l, t, x, p)$ is concave in $l$ then $R(l, t)$ and $W(l, t)$ are also concave in $l$ since if $g(x, y)$ is concave in $y$ the integral $\int g(x, y) d x$ is also concave in $y$.

We prove the result by induction on $n$ the iteration value in equations (8) to (10). The result holds for $n=0$ since $V_{0}(l, t, x, p)=R_{0}(l, t)=W_{0}(l, t)=0$. Assume the hypotheses hold for $V_{n}(l, t, x, p)=R_{n}(l, t)=W_{n}(l, t)$ then since

$$
\max \left\{a_{1}-b_{1}, a_{2}-b_{2}\right\} \geq \max \left\{a_{1}, a_{2}\right\}-\max \left\{b_{1}, b_{2}\right\} \geq \min \left\{a_{1}-b_{1}, a_{2}-b_{2}\right\}
$$

One has

$$
\begin{array}{r}
\quad V_{n+1}\left(L-m x_{0}, t, x_{0}, p\right)-V_{n+1}\left(L-(m+1) x_{0}, t, x_{0}, p\right) \\
\geq \min \left\{R_{n}\left(L-m x_{0}, t\right)-R_{n}\left(L-(m+1) x_{0}, t\right) ; R_{n}\left(L-(m+1) x_{0}, t\right)\right. \\
\left.-R_{n}\left(L-(m+2) x_{0}, t\right)\right\}=R_{n}\left(L-m x_{0}, t\right)-R_{n}\left(L-(m+1) x_{0}, t\right)
\end{array}
$$




$$
\begin{aligned}
& =\max \left\{R_{n}\left(L-(m-1) x_{0}, t\right)-R_{n}\left(L-m x_{0}, t\right) ; R_{n}\left(L-m x_{0}, t\right)-R_{n}(L-(m+\right. \\
& \left.\left.1) x_{0}, t\right)\right\} \geq V_{n+1}\left(L-(m-1) x_{0}, t, x_{0}, p\right)-V_{n+1}\left(L-m x_{0}, t, x_{0}, p\right)
\end{aligned}
$$

where the middle equalities hold because the induction hypotheses gives the concavity of $R_{n}(l, t)$ in $l$. So the induction holds and $V_{n+1}(l, \ldots, .$.$) is concave in l$. Hence so are $R_{n+l}(l,$.$) and W_{n+l}(l,$.$) and also the limit functions of the iteration scheme, V(l, ., .$.$) ,$ $W(l,$.$) and R(l,$.$) .$

ii) It is enough to show $p^{*}\left(L-m x_{0}, t, x_{0}\right) \leq p^{*}\left(L-(m+1) x_{0}, t, x_{0}\right)$

$$
\begin{aligned}
& p^{*}\left(L-m x_{0}, t, x_{0}\right)=\frac{1}{S+F}\left(\frac{R\left(L-m x_{0}, t\right)-R\left(L-(m+1) x_{0}, t\right)}{x_{0}}+F\right) \leq \\
& \frac{1}{S+F}\left(\frac{R\left(L-(m+1) x_{0}, t\right)-R\left(L-(m+2) x_{0}, t\right)}{x_{0}}+F\right)=p^{*}\left(L-(m+1) x_{0}, t, x_{0}\right)
\end{aligned}
$$

Where the inequality follows from the concavity proved in Theorem 2 (i).

In the general case when the borrowers can request different loan amounts, Theorem 2 fails. The problem can be seen if one thinks of the decision whether to give a loan of $2 x_{0}$ as consisting of whether to accept the first $x_{0}$ and then subsequently accept the extra $x_{0}$. One would accept the first if $p \geq p^{*}\left(L-m x_{0}, t, x_{0}\right)$ but only accept the second if $\geq$ $p^{*}\left(L-(m+1) x_{0}, t, x_{0}\right)$ which may not hold. In that case should one reject or accept the loan. Under both decisions one loses the concavity of the $R(l,$.$) function.$

There are two obvious suboptimal policies that could be considered for this problem. The first is the optimal uncapped policy where the lender ignores the cap on lending completely and accepts a borrower with probability $p$ of paying off a loan of $x$ provided:

$$
p \geq p^{U}(i, t, x)=\frac{F}{S+F}
$$

Note $p^{*}(l, t, x) \geq p^{U}(l, t, x)$ so the uncapped policy is less strict than the optimal capped policy. The uncapped policy is suboptimal because it can run out of capital while there is time for more "good" borrowers to appear. 
Another obvious suboptimal policy seeks to just use up all the capital under the assumption that the future is deterministic. Let $\bar{x}$ be the expected value of a loan requested by future borrowers. So

$$
\bar{x}=\int_{0}^{\infty} x d x \int_{0}^{1} f(x, p) d p
$$

Let $H(q)$ be the cumulative probability that a borrower will have a probability of $q$ or less of repaying the loan. So

$$
H(q)=\int_{0}^{q} d p \int_{0}^{\infty} f(x, p) d x
$$

If there is $l$ capital remaining and a time $t$ until the cap horizon is reached then the deterministic future heuristic accepts a loan of $x$ from a borrower with probability $p$ of repaying the loan provided $p \geq \max \left\{p^{D}(l, t, x), p+p^{U}(l, t, x)\right\}$ where

$$
l-x=\lambda t \bar{x}\left(1-H\left(p^{D}\right)\right)
$$

The RHS of this expression is the expected number of future applicants multiplied by the average loan requested multiplied by the probability they will be accepted if the existing decision continues to be applied. The LHS is the capital available if this loan is accepted. So this is set equal to the capital that would be used up if the current policy continued to be used in this deterministic future. However, sometimes there is so much capital this equality means the lender will take unprofitable customers, hence the need to take the maximum of this probability and that of the uncapped case, which takes only the profitable customers. The heuristic is sub optimal because it assumes the future decisions will be the same as the current one. In the next section numerical examples are used to investigate how sub optimal it is.

\section{Discrete Time Approximation to the Lending Model}

Puterman (1994) outlines three ways of solving continuous time Markov decision processes. One can discretize time, use a semi Markov approach or apply uniformization of the Markov process by introducing decisions at times chosen by an independent Poisson process. The latter two approaches are not appropriate for this problem since the time of the decision until the end of the lending horizon is part of the state space. So the time until the end of the lending horizon $T \geq t \geq 0$ is discretized into $N+1$ decision points $(N / N) T,(N-$ 
1)/N)T, $((N-2) / N) T, \ldots .((N-k) / N) T, \ldots((N-N) / N) T$. The chance no applicant appears between one time point and the next is $1-\mu$ where

$$
1-\mu=e^{-\frac{-\lambda T}{N}} \approx\left(1-\frac{\lambda T}{N}+o\left(1 / N^{2}\right)\right)=1-\frac{\lambda T}{N}
$$

So $\mu=\lambda T / N$ is the chance an applicant arrives in that period. One can always choose $N$ large enough that the chance of two or more applicants appearing in the same discrete time interval is negligible.

The $l$ and $x$ parts of $(l, t, x, p)$ can be discretized by assuming there are only a finite number of loan amounts that can be offered. If one takes $x_{0}$ to be the greatest common denominator of these amounts, then the loan amounts can at most be $x_{0}, 2 x_{0}, 3 x_{0}, \ldots, j x_{0}, \ldots, J x_{0}$ where $J=[L / x 0]$. The amount of capital still available must then be $L-m x_{0}$ where $m=$ $0,1,2, \ldots, M$. where $M=\left[\frac{L}{x_{0}}+1\right]$. The probability $p$ of the lender repaying in full is also discrete since it is given by a credit scorecard which can only take a discrete number of values. We let the corresponding probabilities be $p_{1}, p_{2}, \ldots, p_{r}, \ldots, p_{R}$. The probability that an applicant arrives with success probability $p_{r}$ and asks for loan amounts $j x_{0}$ is $p\left(j, p_{r}\right)$. In our examples, we assume $j$ and $p$ are independent. With these assumptions we can modify the optimality equation given in equations (1) to (3) into the following calculation process. This involves rewriting $V\left(L-m x_{0},(N-k) T / N, j x_{0}, p_{r}\right)$ as $V(m, k, j, r)$ and $R(L-$ $\left.m x_{0}, k T / N\right)$ as $V(m, k, 0,$.$) . This latter definition follows because there being no applicant$ at a given time is the same as there being an applicant for a loan of zero. In such a case the probability of repaying is not important. The optimality equation then reduces to the following

$$
\begin{aligned}
& V(m, k, j, r) \\
& =\max \left\{\begin{array}{c}
(1-\mu) V(m, k+1,0, .)+\mu \sum_{j^{\prime}, r^{\prime}} p\left(j^{\prime}, r^{\prime}\right) V\left(m, k+1, j^{\prime}, r^{\prime}\right) \\
j x_{0}\left(S p_{r}-F\left(1-p_{r}\right)+(1-\mu) V(m+j, k+1,0, .)+\mu \sum_{j^{\prime}, r^{\prime}} p\left(j^{\prime}, r^{\prime}\right) V\left(m+j, k+1, j^{\prime}, r^{\prime}\right)\right.
\end{array}\right\}
\end{aligned}
$$

This reduces in the case $j=0$ to 


$$
V(m, k, 0, .)=(1-\mu) V(m, k+1,0, .)+\mu \sum_{j^{\prime}, r^{\prime}} p\left(j^{\prime}, r^{\prime}\right) V\left(m, k+1, j^{\prime}, r^{\prime}\right)
$$

The boundary conditions are $V(m, N, j, r)=V(M, k, j, r)=0$ for all $j, r$.

We can apply this calculation scheme to find $R(l, T)$ for several values of $l$ and $p^{*}(l, t, x)$ for several combinations of $l, t$ and $x$ with several choices of parameters.

\subsection{Numeric Examples}

\section{Example 1}

The following are parameters used in our numeric examples:

$L=M=10 ; N=T=12 ; J=2 ; x_{0}=1 ; \mu=0.5 ;$

$R=6 ;\left[p_{1}, p_{2}, \ldots, p_{r}\right]=[0.7,0.8,0.85,0.9,0.95,0.99] ; p\left(j, p_{r}\right)=\frac{1}{12}, \forall j, r ;$

$r=0.2 ; r_{F}=0.1 ; l_{D}=0.5$;

Using (1), this gives $S=0.1$ and $F=0.6$.

The results for the numerical examples for fully funded, partially funded and not funded are shown in Figure 1, 2 and 3 respectively. In these figures, the $\mathrm{x}$-axes represent the time $k$, where the higher the $k$ the closer it is to the end of the planning horizon. The y-axes represent the optimal cut-off value $p^{*}$ of the corresponding $m, k, j$. In each of these graphs, there are two curves which indicate the cut-off value corresponding to $j=1,2$ respectively. For example, the optimal cut-off for $p^{*}(0,0,1)$ is 0.85715 as shown in Figure 1a. This means if the lender has not lent any money yet $(m=0)$ and it is at the beginning of the planning horizon $(k=0)$, s/he will lend to a borrower, who asks for 1 unit of money $(x=1)$, only if this borrower has a credit score higher than 0.85715 . This is the probability of being Good which means the borrower is expected to be profitable. It is the cut-off value for all borrowers in the unrestricted case.

[Figure 1 about here]

In Figure 1a (where $m=0$ indicates all capital is available), the optimal cut-off values $p^{*}$ for all $j=1,2$ throughout the whole planning horizon are almost identical and are close to the unconstrained cut-off value. When the lender has lent out $m=5$ (shown in Figure 1b), the 
cut-off values are higher than those of $m=0$ because there is less capital available and so the lender needs to be more discriminating. Also, in Figure $1 \mathrm{~b}$, the cut-off values $p^{*}$ decreases with $k$. This makes sense since the lender is running out of time and so needs to accept riskier borrowers. As might be expected, most of the time the cut-off probability $p^{*}(m, k, j)$ decreases with $m$ and decreases as $k$ increases. The lender needs to be more relaxed in whom to accept if they are well below the cap or they have little time left. They never though accept borrowers who are not profitable $(p<0.85715)$. The cut-off probabilities tend to be concave functions of the time since the start of the period. One extra unit of time makes a larger difference to the cut-off probabilities when there is little time left than when it is a long time to the time horizon. Another observation for Figure $1 \mathrm{a}$ and $1 \mathrm{~b}$ is that the cut-off value for $j=$ 1 is lower than those of $j=2$. This means the model favours those borrowing lower amount. $p^{*}(m, k, j)$ varies from the profitable limit of 0.85715 up to almost 1.00 when there is little capital and lots of time available. This is quite a major level of unfairness especially when compared with the uncapped policy which shows the same cut-off probability - 0.85715 - for everyone.

When $m=9$ (shown in Figure 1d), the cut-off for $j=2$ is much lower than those of $j=1$. This is the lender taking advantage that full funding allows him to "overshoot" the capital cap and lend 11 units not just 10 in this case. This leads to a larger profit. When $m=8$ (shown in Figure 1c), it is more favourable to lend money to borrower who asked for $j=1$ than those who wanted $j=2$. With $m=8$, the lender will use up all the capital immediately if it gives credit to those asking for $j=2$. But accepting those with $j=1$ allows the possibility of overshooting in the next period.

[Figure 2 and 3 about here]

For the partially funded and no funded cases (shown in Figure 2 and 3 respectively), the optimal cut-off values for a low $m$ are very similar to those of fully funded cases. However, as $m$ approaches the cap on the capital $(M=12)$, the optimal cut-off for no funded are the highest, following by the partially funded and the fully funded cases have the lowest cut-off value. This is because, including the overshoot capital, the fully funded cases have slightly more capital which essentially leads to a more lax policy. Comparing the partially funded and the no funded cases, since the later has much less opportunities, it tends to be more careful. 
So for example at $m=8$ and $k=0$, the partially funded case accepts borrowers provided their probability of being Good is around 0.95 while in the no funding case, the lender should only accept $j=1$ borrowers if their Good probability is close to 1 . This is partly because in the no funding case the lender cannot accept any one asking for a loan of $j=2$ if they only have 1 unit left, so do not like having only one unit of capital available.

In terms of profitability we compare the expected profits $R(l, t)$ whose discrete time equivalent is $V(m, k, 0,$.$) (which corresponds to R(M-m, N-k)$ ) relative to the optimal uncapped profit. The latter is

$$
(N-k) \mu \sum_{r=1}^{R}(j) p\left(j, p_{r}\right) \max \left\{0,(S+F) p_{r}-F\right\}
$$

Using the parameters in our example, the above equals to $((12-k)(0.5)) / 4\{0+0+0+$ $(.7(0.99)-.6)+(.7(0.95)-.6)+(.7(0.9)-.6)\}=0.0235(12-k)$. In Table 1, we show the uncapped profits as $k$ varies and the capped profits as both $k$ and $m$ vary. If there is plenty of time (12 units) and plenty of capital (10 units), the capped profits are almost the same as the uncapped ones. However, they drop down to well under $50 \%$ as the amount of capital available drops. Note that since the expected profit from 1 unit of capital seems to be a maximum of $S=0.1$ if the borrower has $p=1$, the expected profit if there is only one unit of capital available exceeds this. This is because of the overshooting effect where the lender waits for a Good borrower with high $p$ who needs 2 units to borrow before lending the 1 unit. Table 1 can be thought of as the loss in profit due to capping but also the errors if one uses the uncapped policy to approximate the capped profits.

[Table 1 about here]

The second approximation to the optimal policy is the deterministic future heuristic given by (17). The deterministic time version of this heuristic is

$$
M-(m+j) x_{0}=\frac{\mu(N-k) T \bar{x}}{N}\left(1-H\left(p^{D}\right)\right) \text { with } p^{*}=\max \left\{p^{U}, p^{D}\right\}
$$

Using the parameters of Example 1, this translates to 


$$
p^{*}(m, k, j)=\left\{\begin{array}{c}
0.989 \text { if } 10-(m+j) \leq 0.125(12-k)) \\
0.949 \text { if } 0.125(12-k)<10-(m+j) \leq 0.25(12-k) \\
0.899 \text { if } 0.25(12-k)<10-(m+j) \leq 0.375(12-k) \\
0.8571 \text { if } 0.375(12-k)<10-(m+j)
\end{array}\right\}
$$

The corresponding profits for this policy compares with the optimal uncapped policy is given in Table 2.

\section{[Table 2 about here]}

From Table 2, we see this heuristic has values close to those of the optimal policy when $k>$ $2 m$ but they drop below the optimal policy values when $m$ is large. The former shows that when there is less time $(12-k)$ available than capital $(10-m)$ available the heuristic is essentially optimal. The latter suggests that when there is little capital available it is less accurate. This follows since the LHS of (17) which defines the heuristic is $l-x$ which suggests the loan is granted. If one assumed the loan was not granted the LHS of (17) should be $l$. This clearly matters when $l$, the capital available, is small.

\section{Example 2}

Theorem 1 showed that if all borrowers required the same amount then the cut-off probabilities increased as the capital available increased. We promised to give a counter example to this in the general case and Example 2 is such a counter example. The parameters in this case are

$L=M=4 ; N=T=3 ; J=2 ; x_{0}=1 ; \mu=0.5 ;$

$R=6 ;\left[p_{1}, p_{2}, \ldots, p_{r}\right]=[0.7,0.8,0.85,0.9,0.95,0.99]$;

$p\left(1, p_{r}\right)=\frac{0.01}{6}, p\left(2, p_{r}\right)=\frac{0.99}{6}, \forall r$;

$r=0.2 ; r_{F}=0.1 ; l_{D}=0.5 ; S=0.1$ and $F=0.6$.

Figure 4 shows that for both $k=0$ and 1 , as $m$ increases, the cut-off probabilities $p^{*}(m, 0,1)$ and $p^{*}(m, 1,1)$ are non-monotonic. This is because it is much more likely for borrowers to request a loan of 2 rather 1 . So the lender prefers to have an even amount of capital in the no funding case (and an odd amount in the fully funding case). When a borrower wanting 1 unit applies the lender is more relaxed if he has 1 left (i.e. $m=2$ ) rather when he has 2 left (i.e. $m=1)$. This reasoning also explains why $p^{*}(2,0,1)<p^{*}(2,0,2)$ whereas $p^{*}(1,0,1)>$ 
$p^{*}(1,0,2)$, so that sometimes the lender should be more conservative with loans of 1 and other times with loans of 2 .

[Figure 4 about here]

\section{Lending Decision with Floor on Total Capital Lent}

Whereas the regulators in some countries have put caps on the annual lending to some of the banks' retail portfolios, other countries' regulators have been putting floors on the amount a bank should lend to a particular portfolio. One example would be the Project Merlin proposals started by the UK government in 2011 which put a floor particularly on a bank's lending to SMEs. Models when there is a floor on the amount to be lent in a given period are related to the lending models when there are caps on lending as in the model of Section 3. To see this, suppose the lender must lend at least $L$ of the resources in a time horizon $T$. Applicants arrive according to a Poisson process with parameter $\lambda$. An applicant is described by two parameters $-x$ the amount of the resource (the loan) they require and their probability $p$ of paying back the loan. The distribution of these values over all the applicants is given by identical independent distributions with density function $f(x, p)$.

Let $V(l, t, x, p)$ be the expected future profit to the lender if there is a current applicant with loan size $x$ and probability of repayment of $p$, and the lender is still $l$ below the resource floor ( i.e. $L-l$ has been lent so far) and there is $t$ until the end of the lending horizon. $R(l, t)$ is the expected future profit if there is currently no applicant wanting a loan with the lending still $l$ below the lending floor and $t$ until the end of the lending horizon. Similarly, $W(l, t)$ is the expected future profit if there is an applicant waiting but his characteristics $x$ and $p$ are not yet known and the lending is $l$ below the lending floor and there is $t$ until the end of the lending horizon. Note that $l$ will be in the range $[-\infty, L]$ where a negative value means the lending is already above the floor value.

The optimality equation for these future expected values satisfies 


$$
\begin{aligned}
& V(l, t, x, p)=\max \left\{\begin{array}{c}
R(l, t): \text { reject } \\
x(S p-(1-p) F)+R(l-x, t): \text { acccept }
\end{array}\right. \\
& \text { where } R(l, t)=\int_{0}^{t} \lambda e^{-\lambda s} W(l, t-s) d s(23) \\
& \text { and } \mathrm{W}(l, t)=\int_{0}^{1} d p \int_{0}^{\infty} d x f(x, p) V(l, t, x, p)(24) \\
& \text { with the boundary condition } \mathrm{R}(1,0)=-\mathrm{Ml} \text { if } \mathrm{l}>0 ;=0 \text { if } 1 \leq 0
\end{aligned}
$$

Where $\mathrm{M}$ is the penalty imposed if the lending floor is not reached. This is the same optimality equations as (1) to (3) except for the boundary condition. So with one exception the value functions are non-increasing in $l$ - we get the same results as Lemma 1 . Hence an optimal policy similar to that in Theorem 1 . This can be written as

Lemma 2: $V(l, t, x, p), R(l, t)$ and $W(l, t)$ are all non-increasing in 1 and non-decreasing in $t$, $V(l, t, x, p)$ is non-decreasing in $p$.

Proof. The proof follows Lemma 1 except that $R(I, 0)$ is non-increasing in $l$ and this condition goes through the induction hypothesis.

The next theorem looks at the form of the optimal policy.

Theorem 3: The optimal policy is such that in state $(l, t, x, p)$ one accepts an applicant if

$$
p \geq p_{F}^{*}(l, t, x)=\left\{\begin{array}{cc}
\frac{F+\frac{R(l, t(-R(l-x, t)}{x}}{S+F}=p^{U}-\frac{R(l-x 0, t)-R(l, t)}{(S+F) x} l>0 \\
p^{U}=\frac{S}{(S+F)} & l \leq 0
\end{array}\right\}
$$

Proof. The result for $l>0$ follows directly from (22). For $l \leq 0$ the floor restriction is satisfied and so this is equivalent to the uncapped situation.

Note that as $R(l, t)$ is non-increasing in $l, p_{F}^{*}(l, t, x) \leq p^{U}$ and so the lender needs to take riskier applicants than the uncapped case until the floor is reached.

\section{Example 3}

For this lending example with a floor in the lending the parameters are the same as those of Example 1. For the boundary condition, we assume $M=-10 l$, i.e. a penalty of 10 for every unit of capital that the lending is below the floor requirement. In the floor model, the lender 
either does not fund an applicant or funds them fully as there is no upper restriction on the amount of capital to lend. Note that from Theorem 3, the lender is more relaxed in his policy while he is below the floor compared with the unconstrained case. Figure 5 describes the cutoff probabilities as a function of $k$ the time since the start of the time horizon. Surprisingly this looks almost like a bang-bang policy swapping between taking only profitable borrowers and taking all borrowers. As $k$ increases and so there is less time available the cut-off decreases since the lender is more and more concerned about reaching the floor. Eventually this condition is so important the lender takes everyone. This desperate decision occurs earlier and earlier the less capital that has been already lent. At $m=0$ the lender takes everyone from $k=0$; for $m=5$ it occurs 3 periods from the start and with $m=9$ and so only one more unit of capital to meet the floor it is not until period 10.

\section{[Figure 5 about here]}

The probability cut-off is of the bang-bang type as $k$ varies both for loans of value $x_{0}$ and $2 x_{0}$. In the region as one moves from one extreme to the other the lender is more cautious with larger loans than smaller ones. This is because it is now likely the lender will reach his floor target and so is getting more concerned about the losses he is incurring on these loans.

In this floor case, one needs to do a little more work to find the optimal expected profitability than just take $V(m, k, 0,$.$) That value will contain the penalty for not reaching the floor in$ some cases. One needs to take the optimal policy obtained from the optimal equations and rerun the calculations allowing only those optimal actions and removing the boundary penalty for failing to meet the cap. The results of doing this compared with the profit from the capped policy are shown in Table 3.

\section{[Table 3 about here]}

Table 3 shows that imposing floors has a higher impact on profit than imposing caps. When a lender is still a long way from the floor the effect on his profit is very severe, sometime he only gets $1 \%$ of the optimal uncapped profit. $18 \%$ in the example under consideration corresponds to the policy of taking every applicant. Doing so in order to meet the floor has a major impact on profitability. Profits only start to recover to the uncapped case when $m$ is high and $\mathrm{k}$ small. This means the lender has almost reached the floor and still has a long time to go before the time horizon. 


\section{Other Non-lending Examples}

This variant of the multiple choice secretary problem or the polygamous marriage problem can occur in several other contexts than consumer lending. Some examples are as follows.

\section{Anti-missile ship defence}

A warship is provided with $\mathrm{L}$ anti-missile missiles for ship defence. It believes it can come under missile attack and not be able to refurnish with anti-missile missiles for a period $\mathrm{T}$. Attacking missiles are likely to arrive in a Poisson process with parameter $\lambda$. Each attacking missile is described by two parameters, $y$ the damage it can cause if it hits the ship and $p$ the probability that the anti missile will be able to destroy it. Take $x=1, S=0$ and $F=-y$. The objective is to minimise the damage to the ship, The corresponding model is a Markov decision process given by equations (1) to (3) with $y$ added to the state space if necessary so that in state $(l, t, y, p)$ there are $l$ anti-missile missiles remaining, $t$ until the end of the attack and the current attacking missile is described by parameters $y$ and $p$.

\section{Refugee Acceptance policy}

How many and which refugees should a country accept after a natural catastrophe or internal civil was in a neighbouring territory is an important humanitarian decision in current times. The problem is whether the refugees will suffer persecution (civil war) or starvation (natural catastrophe) if they are not allowed into the country. Let $L$ be the number of refugees that will be allowed in over a period $t$. Assume the refugees arrive according to a Poisson process with parameter $\lambda$. Each refugee family has two characteristics $-x$ the size of the family and $p$ the probability they will suffer persecution or hunger if they are not allowed in. Let $S=1, F=0$ and the objective is to maximise the expected numbers who avoid hunger or persecution by being accepted as refugees. This can be modelled as a Markov decision process and its optimality equation coincides with (1) to (3).

\section{Inoculation Campaign}

One of the most successful ways of dealing with diseases like influenza, pneumonia or measles is by inoculation against the disease. If there is a new or unexpected outbreak of the disease then there will be a limit $L$ on the number of vaccine shots available. It is expected the outbreak will last a time $T$ and patients appear for inoculation during this period 
according to a Poisson process with parameter $\lambda$. Each patient is assessed to estimate $p$ the probability of them getting the disease if not inoculated. Let $x=1, S=1, F=0$. The health authority wishes to maximise the expected number of patients who have been prevented from getting the disease by the inoculation campaign. The corresponding Markov Decision Process model leads to the same optimality equation as is found in (1) to (3).

\section{Conclusion}

Imposing floors and caps on the amount of capital a bank can lend in a given period is one way financial regulators are controlling the economy. This paper looks at the resultant decision that a bank has to make on which loan applicants to accept and which to reject. From these, we can identify the inefficiencies and unfairness such constraints cause.

The decision is modelled as a continuous time Markov Decision Process. Doing so highlights several issues. Firstly is the cap an absolute or flexible limit on what is lent? Depending on which situation, one has three cases at the cap limit of fully funding, partially funding or not funding a loan which takes the bank over the cap. Secondly one would expect that having more capital available means the bank will be less restrictive to whom it lends. Surprisingly, this is not the case in general but holds if all the loans are for the same amount. Similarly, one might expect the lender to be more restrictive for a higher amount of loan but again this is not the case. This is because the lending decision is affected by the distribution of loan amount that subsequent applicants may require. Thirdly we can use the model to compare numerically the optimal policy in the uncapped case of only taking profitable applicants with that in the capped case. The results show that capping imposes considerable drops in profit up to $60 \%$ compared with the uncapped case. Also capping makes the accept/reject decision inconsistent with the cut-off probability of being Good in order to be accepted varying from 0.857 to 1.00 even with the small levels of time and capital used in the example.

The floor model also shows that imposing a floor on lending cuts the profitability to the bank by a considerable percentage - up to $82 \%$ in the extreme case. The optimal policy in the floor case is close to a bang-bang one switching between taking everyone and only those who are profitable. If past lending is well below the floor, the lender accepts everyone. With slightly higher levels of previous lending one takes some but not all the unprofitable applicants and all the profitable ones. When past lending is close to the floor or over it, the lender takes only 
profitable applicants. We feel though that this paper addressed the main issues affecting banks decisions to lend when there are floors and caps put on lending over a given time horizon by the financial authorities.

One possible extension is to model the situation where lender can defer decision on whether to offer the loan to later in the lending horizon. This allows lenders to have better knowledge on whether the constraints may be broken or not. However, during the delay time, the borrower may decide to withdrew their application for a loan and apply to a different borrower. A more complicated extension occurs in the credit union area. There the capital available depends on the total deposits of the savers in the credit union which is varying over time. Thus the cap on future lending is both unknown and varying. Addressing this issue will be of important in the future give the prominence that credit union are receiving as part of the debate on unfair lending practices.

\section{References}

Bank of England (2012). Additional data for lending to UK businesses including Project Merlin data. http://www.bankofengland.co.uk/publications/pages/other/monetary/additionaldata.aspx

Bateni, M.H., Hajiaghayi, M.T. \& Zadimoghaddam, M. (2010). Submodular secretary problem and extensions. In M.Serna (Eds.), APPROX and RANDOM 2010 (pp. 39-52). Heidelberg: Springer Verlag.

Bauerle, N., \& Rieder, U. (2011). Markov Decision Processes with Applications in Finance, Berlin: Springer-Verlad.

Bloomberg (2012). Chinese officials said to weigh easing constraints on banks, www.blomberg.com/news/2012-01-18/

Chun, Y. H. (1999). Selecting the best choice in the full information group interview problem. European Journal of Operational Research, 119, 635-651

Derman, C., Lieberman, G.J., \& Ross, M. (1972). A sequential stochastic assignment problem. Management Science, 18, 349-355.

Ferguson, T.S. (1989). Who solved the secretary problem? Statistical Science, 4, 282-296.

Ferguson, T.S., \& Klass, M.J. (2010). House-hunting without second moments. Sequential Analysis: Design Methods and Applications, 29, 236-244 
Freeman, P.R. (1983). The secretary problem and its extensions: a review. International Statistical Review, 51, 189-206.

Kleywegt, N., \& Papastavrou, J.D. (1998). The dynamic and stochastic knapsack problem. Operations Research, 46, 17-35.

Kleywegt, N., \& Papastavrou, J.D. (2001). The dynamic and stochastic knapsack problem with random sized items. Operations Research, 49, 26-41.

Preater, J. (1994). On multiple choice secretary problems. Mathematics of Operations Research, 19, 597-602.

Prastacos, G.P. (1983). Optimal sequential investment decisions under conditions of Uncertainty. Management Science, 29, 118-134.

Puterman, M.L. (1994). Markov Decision Processes. Wiley, New York.

Reserve Bank of India (2009). Credit delivery to the micro and small enterprise sector. $\underline{\text { http://rbi.org.in/scripts/NotificationUser.aspx? } I d=4964 \& M o d e=0}$

Smith, M.H. (1975). A secretary problem with uncertain employment. Journal of Applied Probability, 12, 620-624.

Stein, W. E., Seale, D. A., \& Rapoport, A. (2003). Analysis of heuristic solutions to the best choice problem. European Journal of Operational Research, 151, 140-152

Thomas, L.C. (2009). Consumer Credit Models, Pricing Profit and Portfolios. Oxford University Press, Oxford.

Van Slyke, R., \& Young, W. (2000). Finite horizon stochastic knapsacks with applications to yield management. Operations Research, 48,155-172.

Yoshida, M. (1984). Probability maximising approach to a Secretary Problem with random change-point of the distribution law of the observed process. Journal of Applied Probability, 21, 98-107.

Zhuang, W., Gumus, M., \& Zhang D. (2012). A single-resource revenue management problem with random resource consumptions. Journal of the Operational Research Society, 63, 1213-1227. 


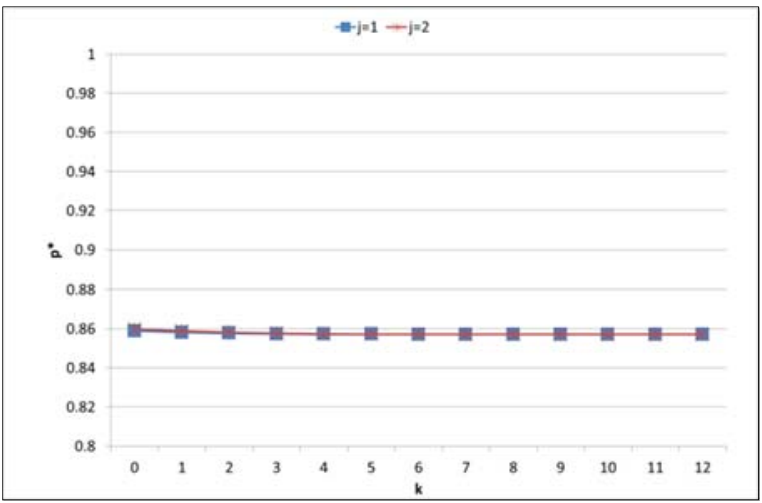

(a.) $p^{*}(0, k, j)$

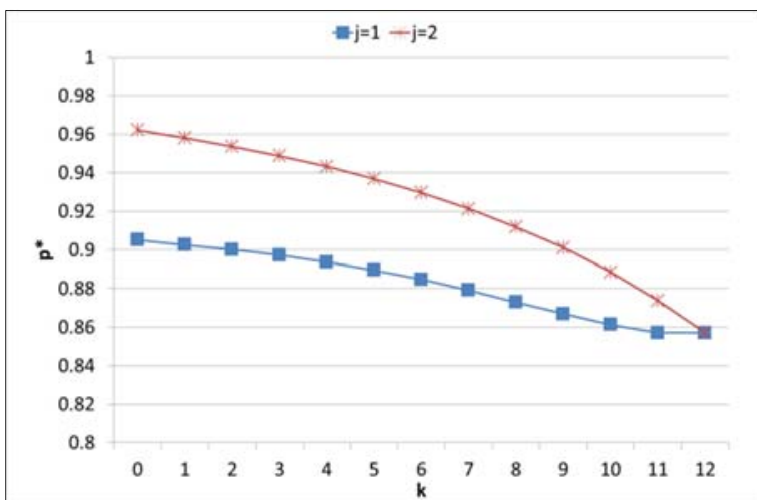

(c.) $p^{*}(8, k, j)$

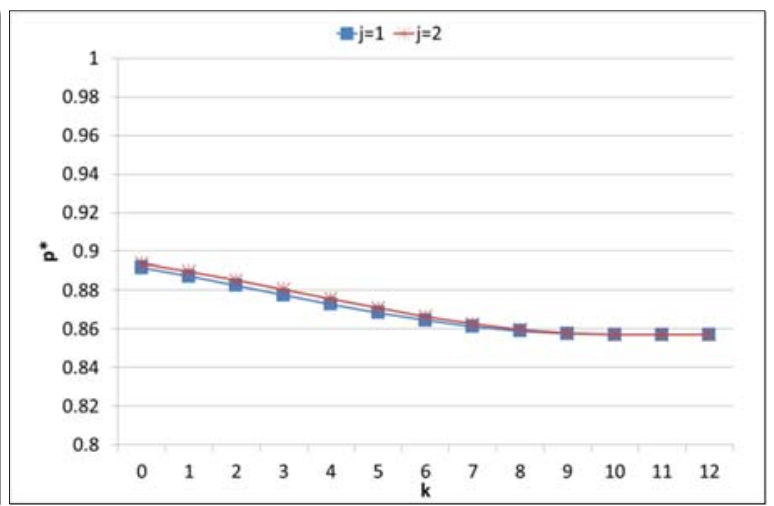

(b.) $p^{*}(5, k, j)$

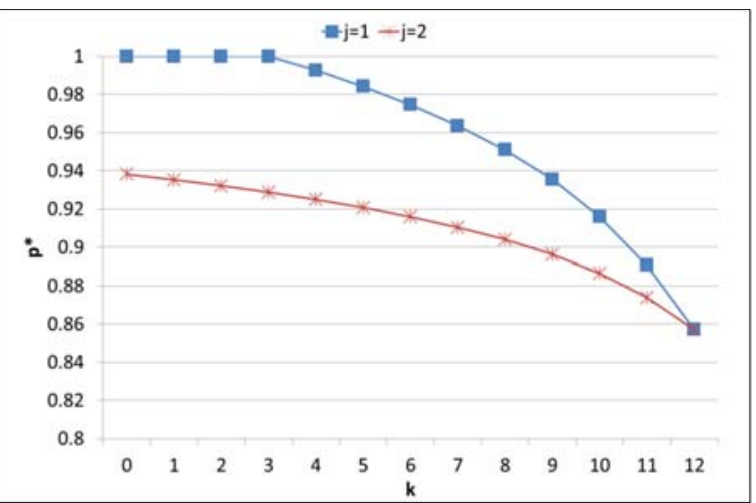

(d.) $p^{*}(9, k, j)$

Figure 1 . The cut-off value $p^{*}$ for Capped fully funded cases.

\begin{tabular}{|c|c|c|c|c|c|c|c|c|c|c|c|c|}
\hline & $\mathbf{k}=\mathbf{0}$ & $\mathbf{k}=\mathbf{1}$ & $\mathbf{k}=\mathbf{2}$ & $\mathbf{k}=\mathbf{3}$ & $\mathbf{k}=\mathbf{4}$ & $\mathbf{k}=\mathbf{5}$ & $\mathbf{k}=\mathbf{6}$ & $\mathbf{k}=\mathbf{7}$ & $\mathbf{k}=\mathbf{8}$ & $\mathbf{k}=\mathbf{9}$ & $\mathbf{k}=\mathbf{1 0}$ & $\mathbf{k}=\mathbf{1 1}$ \\
\hline \begin{tabular}{|} 
Uncappe \\
$\mathbf{d}$ Profit
\end{tabular} & 0.282 & 0.259 & 0.235 & 0.212 & 0.188 & 0.165 & 0.141 & 0.118 & 0.094 & 0.071 & 0.047 & 0.024 \\
\hline
\end{tabular}

Table 1. The difference between Capped (fully funded) and Uncapped for the fully funded case. 


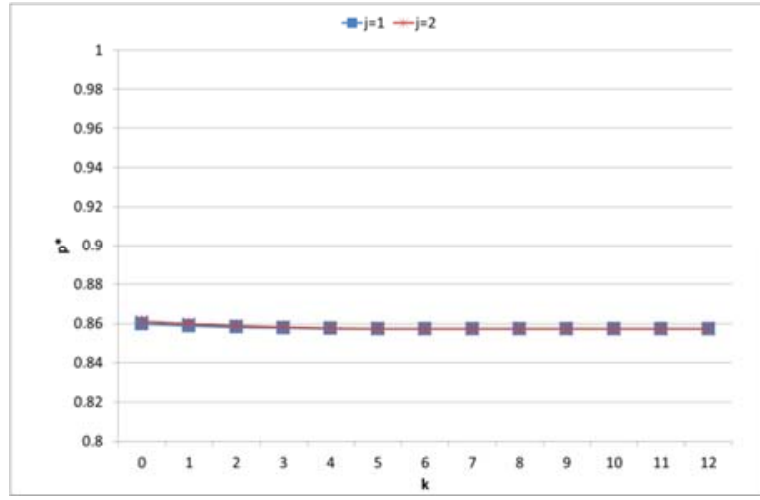

(a.) $p^{*}(0, k, j)$

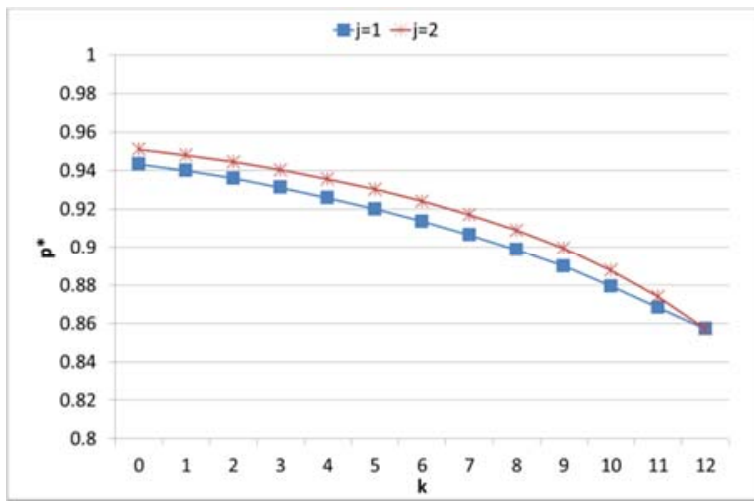

(c.) $p^{*}(8, k, j)$

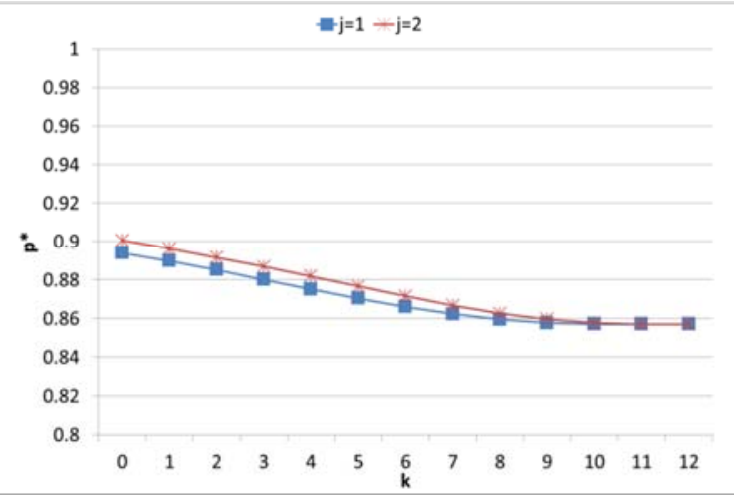

(b.) $p^{*}(5, k, j)$

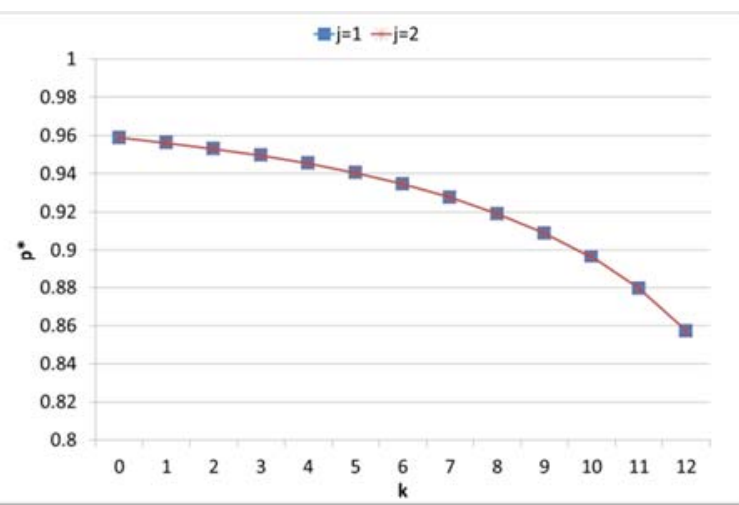

(d.) $p^{*}(9, k, j)$

Figure 2. The cut-off value $p^{*}$ for Capped partially funded cases. 


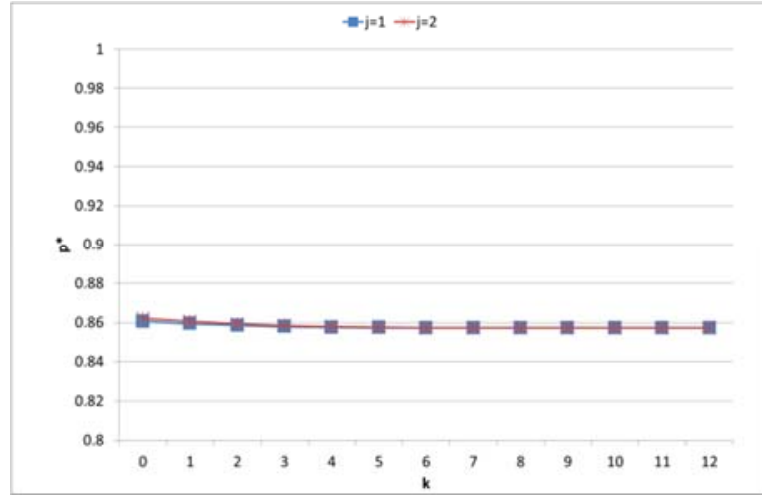

(a.) $p^{*}(0, k, j)$

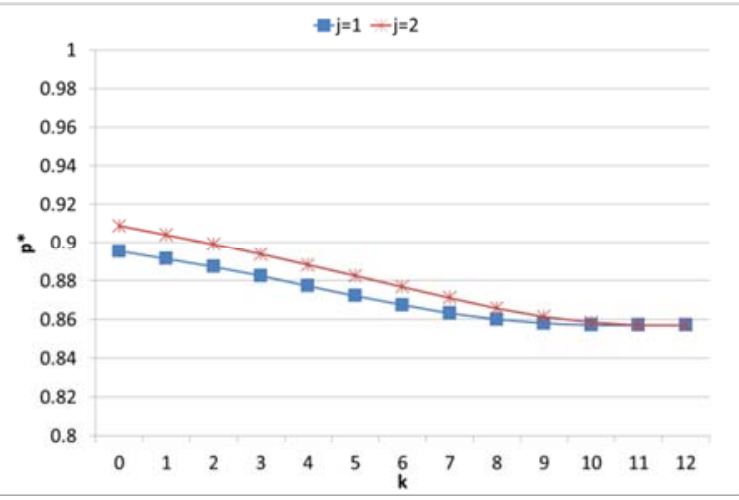

(b.) $p^{*}(5, k, j)$

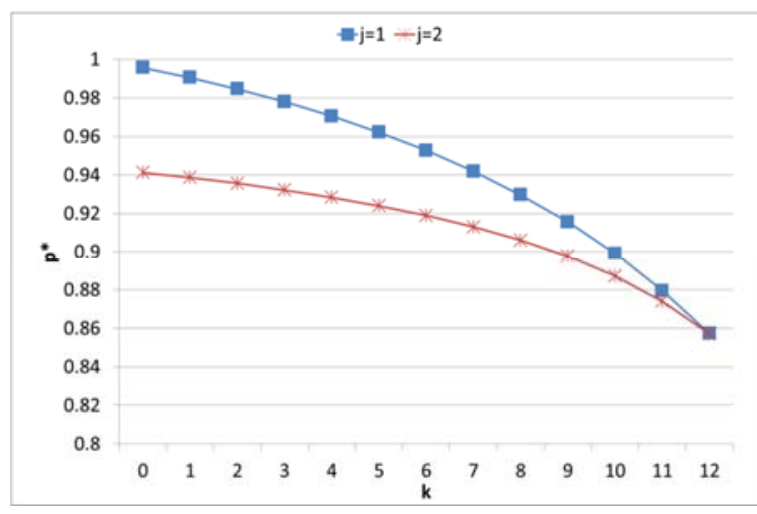

(c.) $p^{*}(8, k, j)$

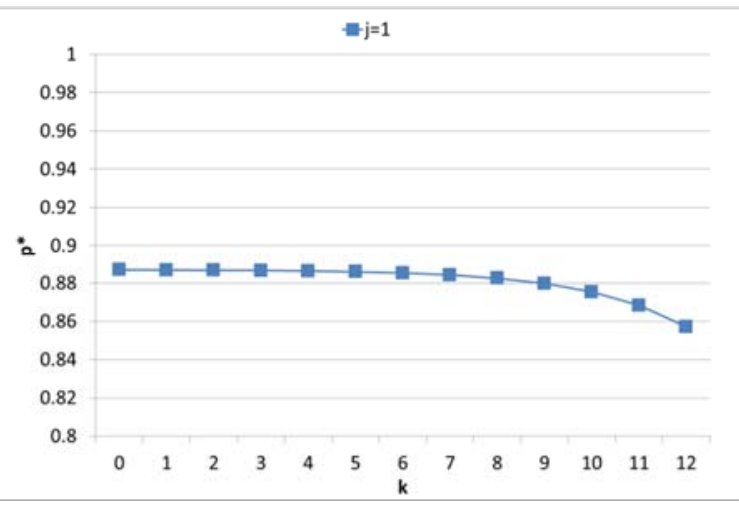

(d.) $p^{*}(9, k, j)$

Figure 3. The cut-off value $p^{*}$ for Capped no funded cases. 


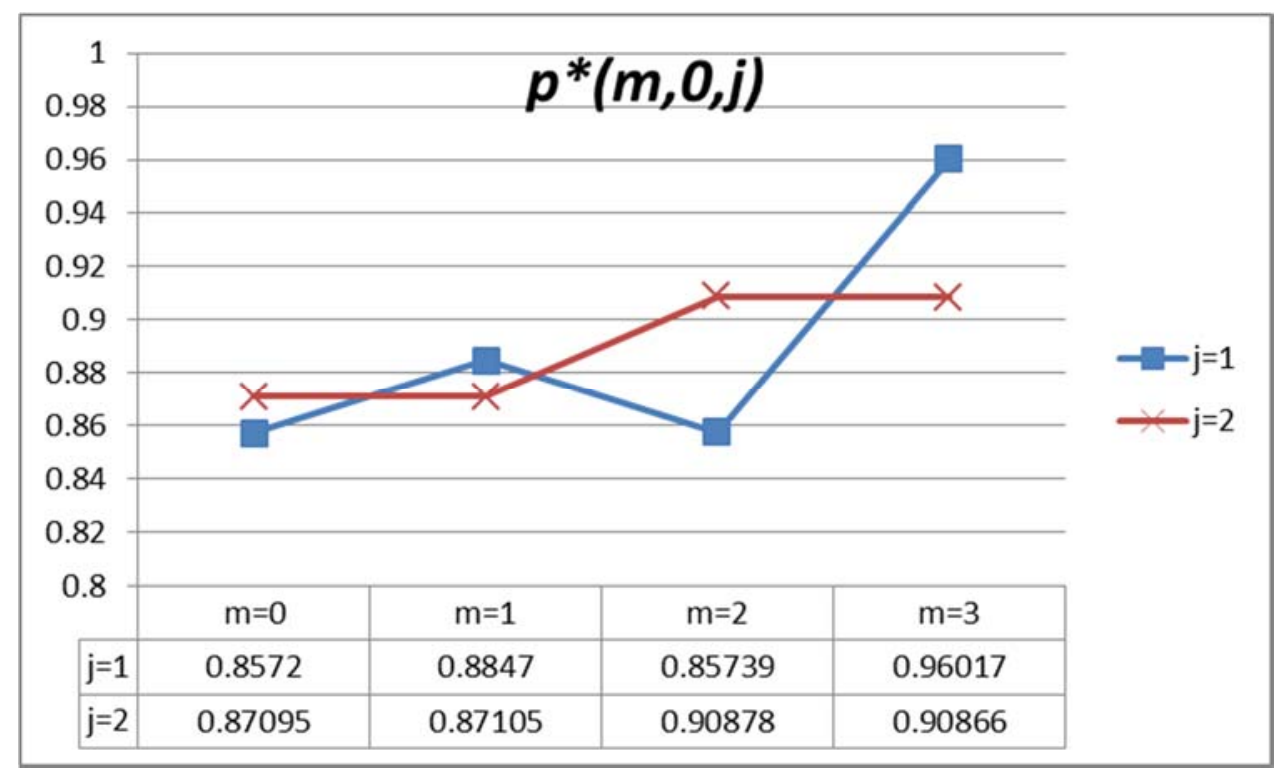

(a.) $p^{*}(m, 0, j)$

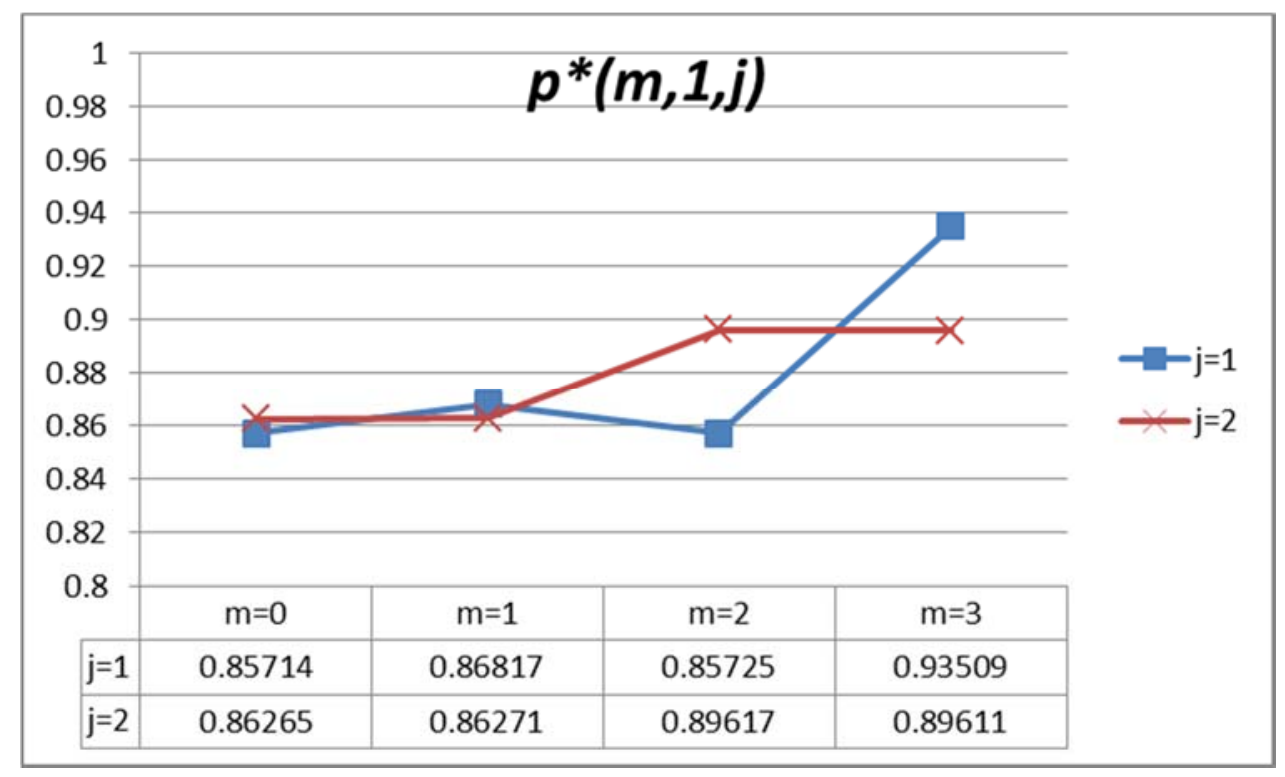

(b.) $p^{*}(m, 1, j)$

Figure 4. A counter example for $p^{*}$ does not increase in $m$. 


\begin{tabular}{|c|c|c|c|c|c|c|c|c|c|c|c|c|}
\hline \multicolumn{13}{|c|}{$\begin{array}{l}\text { Profit of Heuristics in percentage of optimal profit to the corresponding } k \text { and } m \\
\text { (i.e. } \frac{\text { Profit of Heuristic }}{\text { Profit of Capped }} \times 100 \% \text { ) }\end{array}$} \\
\hline & $\mathbf{k}=\mathbf{0}$ & $k=1$ & $k=2$ & $k=3$ & $k=4$ & $k=5$ & $k=6$ & $k=7$ & $k=8$ & $k=9$ & $k=10$ & $k=11$ \\
\hline $\mathbf{m}=\mathbf{0}$ & $99 \%$ & $99 \%$ & $100 \%$ & $100 \%$ & $100 \%$ & $100 \%$ & $100 \%$ & $100 \%$ & $100 \%$ & $100 \%$ & $100 \%$ & $100 \%$ \\
\hline $\mathbf{m}=\mathbf{1}$ & $98 \%$ & $98 \%$ & $99 \%$ & $99 \%$ & $100 \%$ & $100 \%$ & $100 \%$ & $100 \%$ & $100 \%$ & $100 \%$ & $100 \%$ & $100 \%$ \\
\hline $\mathbf{m}=\mathbf{2}$ & $96 \%$ & $97 \%$ & $97 \%$ & $98 \%$ & $99 \%$ & $99 \%$ & $100 \%$ & $100 \%$ & $100 \%$ & $100 \%$ & $100 \%$ & $100 \%$ \\
\hline $\mathbf{m}=\mathbf{3}$ & $92 \%$ & $93 \%$ & $95 \%$ & $96 \%$ & $97 \%$ & $98 \%$ & $99 \%$ & $99 \%$ & $100 \%$ & $100 \%$ & $100 \%$ & $100 \%$ \\
\hline $\mathbf{m}=4$ & $86 \%$ & $88 \%$ & $90 \%$ & $92 \%$ & $94 \%$ & $95 \%$ & $97 \%$ & $98 \%$ & $99 \%$ & $100 \%$ & $100 \%$ & $100 \%$ \\
\hline $\mathbf{m}=5$ & $74 \%$ & $77 \%$ & $81 \%$ & $86 \%$ & $88 \%$ & $90 \%$ & $92 \%$ & $94 \%$ & $96 \%$ & $98 \%$ & $100 \%$ & $100 \%$ \\
\hline$m=6$ & $67 \%$ & $69 \%$ & $71 \%$ & $74 \%$ & $76 \%$ & $79 \%$ & $83 \%$ & $88 \%$ & $91 \%$ & $94 \%$ & $98 \%$ & $100 \%$ \\
\hline $\mathrm{m}=7$ & $69 \%$ & $68 \%$ & $66 \%$ & $65 \%$ & $65 \%$ & $66 \%$ & $68 \%$ & $70 \%$ & $71 \%$ & $74 \%$ & $80 \%$ & $100 \%$ \\
\hline $\mathbf{m}=\mathbf{8}$ & $75 \%$ & $73 \%$ & $72 \%$ & $70 \%$ & $68 \%$ & $66 \%$ & $64 \%$ & $62 \%$ & $61 \%$ & $60 \%$ & $60 \%$ & $66 \%$ \\
\hline $\mathbf{m}=9$ & $80 \%$ & $79 \%$ & $77 \%$ & $76 \%$ & $74 \%$ & $72 \%$ & $69 \%$ & $66 \%$ & $62 \%$ & $58 \%$ & $54 \%$ & $49 \%$ \\
\hline
\end{tabular}

Table 2: Comparison of profits for optimal capped policy and deterministic future heuristic policy 


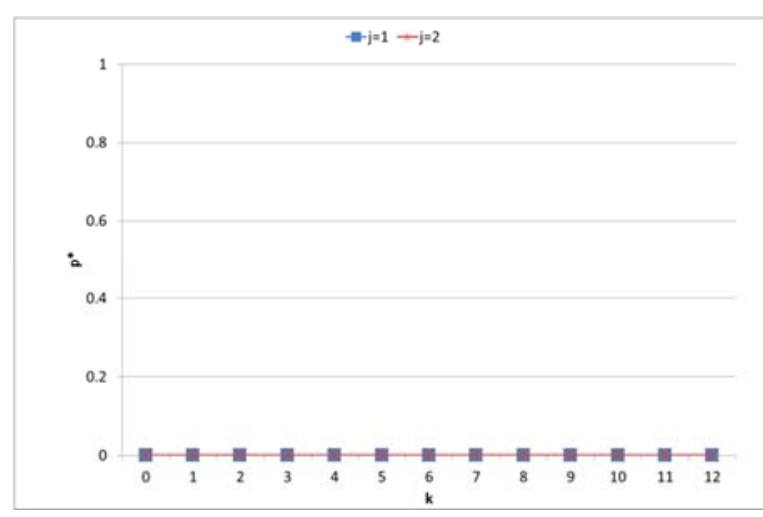

(a.) $p^{*}(0, k, j)$

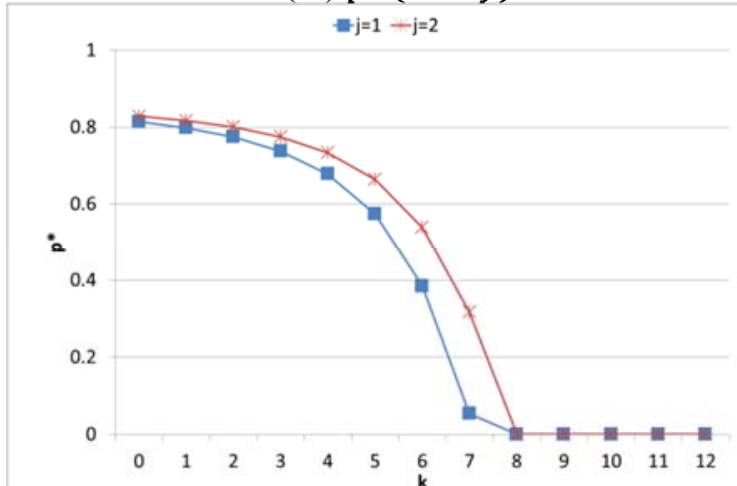

(c.) $p^{*}(8, k, j)$

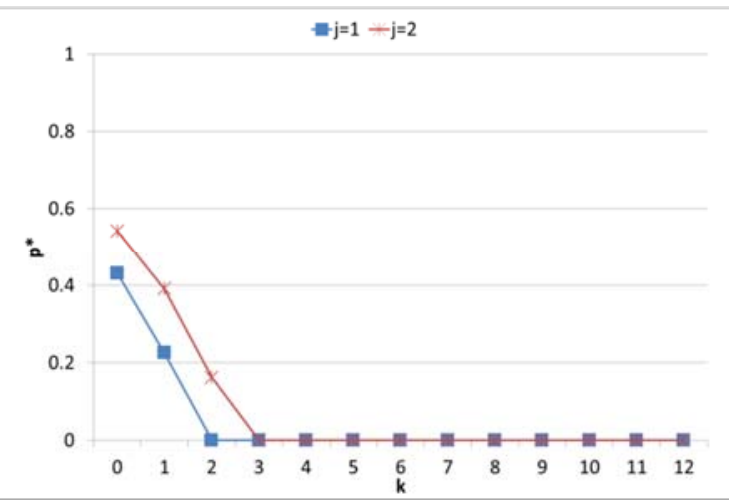

(b.) $p^{*}(5, k, j)$

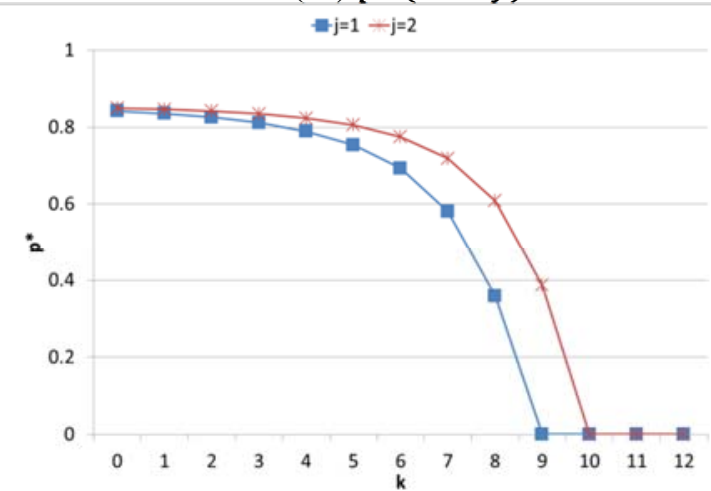

(d.) $p^{*}(9, k, j)$

Figure 5. The cut-off value $p^{*}$ for the example with floor on total capital lent

\begin{tabular}{|c|c|c|c|c|c|c|c|c|c|c|c|c|}
\hline & $k=0$ & $k=1$ & $k=2$ & $k=3$ & $k=4$ & $k=5$ & $k=6$ & $k=7$ & $k=8$ & $k=9$ & $k=10$ & $k=11$ \\
\hline $\begin{array}{l}\text { Uncappe } \\
\text { d Profit }\end{array}$ & 0.282 & 0.259 & 0.235 & 0.212 & 0.188 & 0.165 & 0.141 & 0.118 & 0.094 & 0.071 & 0.047 & 0.024 \\
\hline \multicolumn{13}{|c|}{ Profit in percentage of uncapped profit to the corresponding $\mathrm{k}$ and $\mathrm{m}$ (i.e. $\frac{\text { Prof it of Floor }}{\text { Prof it of Uncapped }} \times 100 \%$ ) } \\
\hline $\mathbf{m}=\mathbf{0}$ & $23 \%$ & $21 \%$ & $20 \%$ & $19 \%$ & $18 \%$ & $18 \%$ & $18 \%$ & $18 \%$ & $18 \%$ & $18 \%$ & $18 \%$ & $18 \%$ \\
\hline $\mathbf{m}=1$ & $27 \%$ & $24 \%$ & $22 \%$ & $20 \%$ & $19 \%$ & $18 \%$ & $18 \%$ & $18 \%$ & $18 \%$ & $18 \%$ & $18 \%$ & $18 \%$ \\
\hline$m=2$ & $32 \%$ & $28 \%$ & $25 \%$ & $22 \%$ & $20 \%$ & $19 \%$ & $18 \%$ & $18 \%$ & $18 \%$ & $18 \%$ & $18 \%$ & $18 \%$ \\
\hline $\mathbf{m}=\mathbf{3}$ & $38 \%$ & $34 \%$ & $30 \%$ & $26 \%$ & $23 \%$ & $21 \%$ & $19 \%$ & $18 \%$ & $18 \%$ & $18 \%$ & $18 \%$ & $18 \%$ \\
\hline$m=4$ & $47 \%$ & $42 \%$ & $37 \%$ & $32 \%$ & $28 \%$ & $24 \%$ & $21 \%$ & $19 \%$ & $18 \%$ & $18 \%$ & $18 \%$ & $18 \%$ \\
\hline $\mathrm{m}=5$ & $57 \%$ & $51 \%$ & $46 \%$ & $40 \%$ & $35 \%$ & $30 \%$ & $26 \%$ & $22 \%$ & $19 \%$ & $18 \%$ & $18 \%$ & $18 \%$ \\
\hline $\mathrm{m}=6$ & $68 \%$ & $62 \%$ & $57 \%$ & $51 \%$ & $45 \%$ & $39 \%$ & $34 \%$ & $28 \%$ & $23 \%$ & $19 \%$ & $18 \%$ & $18 \%$ \\
\hline $\mathrm{m}=7$ & $83 \%$ & $77 \%$ & $69 \%$ & $63 \%$ & $57 \%$ & $51 \%$ & $44 \%$ & $37 \%$ & $30 \%$ & $23 \%$ & $18 \%$ & $18 \%$ \\
\hline $\mathbf{m}=8$ & $93 \%$ & $90 \%$ & $85 \%$ & $79 \%$ & $72 \%$ & $67 \%$ & $61 \%$ & $55 \%$ & $47 \%$ & $38 \%$ & $28 \%$ & $18 \%$ \\
\hline $\mathbf{m}=9$ & $98 \%$ & $97 \%$ & $96 \%$ & $93 \%$ & $90 \%$ & $84 \%$ & $78 \%$ & $68 \%$ & $61 \%$ & $52 \%$ & $38 \%$ & $18 \%$ \\
\hline $\mathrm{m}=10$ & $100 \%$ & $100 \%$ & $100 \%$ & $100 \%$ & $100 \%$ & $100 \%$ & $100 \%$ & $100 \%$ & $100 \%$ & $100 \%$ & $100 \%$ & $100 \%$ \\
\hline
\end{tabular}

Table 3. The difference between Floor and Uncapped (the fully funded case). 